\title{
UNITARINESS OF OPERATORS
}

\author{
C. PADHY, P. K. JENA, AND S. K. PAIKRAY
}

Received 20 June, 2019

\begin{abstract}
In this paper, we explain some sufficient conditions for unitariness of Toeplitz operators and little Hankel operators on the Bergman space.
\end{abstract}

2010 Mathematics Subject Classification: 47B38; 47B35

Keywords: Toeplitz operators, little Hankel operators, unitary operators, Bergman space

\section{INTRODUCTION}

The Bergman space is the Hilbert space of all holomorphic functions $f$ on the open unit disk $\mathbb{D}=\{z \in \mathbb{C}:|z|<1\}$, denoted as $A^{2}(\mathbb{D})$ for which

$$
\|f\|_{A^{2}(\mathbb{D})}=\left(\int\left(|f(z)|^{2} d A(z)\right)\right)^{\frac{1}{2}}<\infty
$$

where $d A(z)$ is the normalized Lebesgue area measure on the open unit disk $\mathbb{D}$. If $h(z)=\sum_{n=0}^{\infty} a_{n} z^{n}$ and $k(z)=\sum_{n=0}^{\infty} b_{n} z^{n}$ are two functions in $A^{2}(\mathbb{D})$, then the inner product of $h$ and $k$ is given by

$$
\langle h, k\rangle=\int_{\mathbb{D}} h(z) \overline{k(z)} d A(z)=\sum_{n=0}^{\infty} \frac{a_{n} \overline{b_{n}}}{n+1} .
$$

The Bergman reproducing kernel is the function $K_{z} \in A^{2}(\mathbb{D})$ for $z \in \mathbb{D}$ such that $f(z)$ $=\left\langle f, K_{z}\right\rangle$ for all $f \in A^{2}(\mathbb{D})$ and normalized reproducing kernel $k_{z}$ is the function $\frac{K_{z}}{\left\|K_{z}\right\|_{2}}$. Here the norm $\|.\|_{2}$ and the inner product $\langle.,$.$\rangle are taken in the space L^{2}(\mathbb{D}, d A)$. For any integer, $n \geq 0$, let $e_{n}(z)=\sqrt{n+1} z^{n}$. Then, $\left\{e_{n}\right\}_{n=0}^{\infty}$ forms an orthonormal basis for $A^{2}(\mathbb{D})$. The Toeplitz operator $T_{\phi}$ with symbol $\phi \in L^{\infty}(\mathbb{D})$ on $A^{2}(\mathbb{D})$ is defined by $T_{\phi} f=P(\phi f)$; here $P$ is an orthogonal projection from $L^{2}(\mathbb{D}, d A)$ onto $A^{2}(\mathbb{D})$.

Let $\overline{A^{2}(\mathbb{D})}$ be the space of conjugate analytic functions in $L^{2}(\mathbb{D}, d A)$. Then $\overline{A^{2}(\mathbb{D})}=$ $\left\{\bar{g}: g \in A^{2}(\mathbb{D})\right\}$ is closed in $L^{2}(\mathbb{D}, d A)$. Let $\phi \in L^{\infty}(\mathbb{D})$, the little Hankel operator $h_{\phi}: A^{2}(\mathbb{D}) \rightarrow A^{2}(\mathbb{D})$ be defined by $h_{\phi} f=\bar{P}(\phi f), f \in A^{2}(\mathbb{D})$ where $\bar{P}$ is the orthogonal projection from $L^{2}\left(\mathbb{D}, d A\right.$ ) onto $\overline{A^{2}(\mathbb{D})}$. There are also numerous equivalent 
ways of defining little Hankel operators on $A^{2}(\mathbb{D})$. For illustration, define the map $S_{\phi}: A^{2}(\mathbb{D}) \rightarrow A^{2}(\mathbb{D})$ by $S_{\phi} f=P J(\phi f)$, where $J$ is selfadjoint, unitary mapping from $L^{2}(\mathbb{D}, d A)$ into itself given by $J h(z)=h(\bar{z})$. Observe that, $J S_{\phi}=h_{\phi}$. So $S_{\phi}$ is unitarily equivalent to $h_{\phi}$.

Let $A u t(\mathbb{D})$ be the Lie group of all automorphisms (biholomorphic mappings) of $\mathbb{D}$. We can define for each $a \in \mathbb{D}$, an automorphism $\phi_{a}$ in $A u t(\mathbb{D})$ such that

(i) $\left(\phi_{a} o \phi_{a}\right)(z) \equiv z$;

(ii) $\phi_{a}(0)=a, \phi_{a}(a)=0$;

(iii) $\phi_{a}$ has a unique fixed point in $\mathbb{D}$.

In fact, $\phi_{a}(z)=\frac{a-z}{1-\bar{a} z}$ for all $a$ and $z$ in $\mathbb{D}$. It is easy to verify that the derivative of $\phi_{a}$ at $z$ is equal to $-k_{a}(z)$. It implies the real Jacobian determinant of $\phi_{a}$ at $z$ is

$$
J_{\phi_{a}(z)}=\left|k_{a}(z)\right|^{2}=\frac{\left(1-|a|^{2}\right)^{2}}{|1-\bar{a} z|^{4}} .
$$

Given $a \in \mathbb{D}$ and $f$ (any measurable function on $\mathbb{D}$ ), let us define a function $U_{a} f$ on $\mathbb{D}$ by $U_{a} f(w)=k_{a}(w) f\left(\phi_{a}(w)\right)$. Notice that $U_{a}$ is a bounded linear operator on $L^{2}(\mathbb{D}, d A)$ and $A^{2}(\mathbb{D})$ for all $a \in \mathbb{D}$. Further, it can be verified that $U_{a}^{2}=I$, the identity operator, $U_{a}^{*}=U_{a}, U_{a}\left(A^{2}(\mathbb{D})\right) \subset A^{2}(\mathbb{D})$ and $U_{a}\left(\left(A^{2}(\mathbb{D})\right)^{\perp}\right) \subset\left(A^{2}(\mathbb{D})\right)^{\perp}$ for all $a \in \mathbb{D}$. Thus $U_{a} P=P U_{a}$ for all $a \in \mathbb{D}$ (see [11]).

Let $H^{\infty}(\mathbb{D})$ denote the space of bounded analytic functions on $\mathbb{D}$. Let $\mathcal{L}(H)$ denote the algebra of bounded, linear operators from a Hilbert space $H$ into itself and let $T \in$ $\mathcal{L}(H)$. Then the Berezin transform of $T$ is denoted by $\widetilde{T}$, a complex valued function on $\mathbb{D}$ defined by $\widetilde{T(z)}=\left\langle T k_{z}, k_{z}\right\rangle$. Let $\operatorname{ker}(T)$ denotes kernel of $T$ and an operator $T$ is normaloid if, $\|T\|=\sup \{|\langle T x, x\rangle| ;\|x\|=1\}$. An operator $T$ is paranormal if, $\|T x\|^{2} \leq\left\|T^{2} x\right\|\|x\|$ and p-hyponormal if $\|T\|^{2 p} \geq\left\|T^{*}\right\|^{2 p}$ for $0<p \leq 1$.

In this article, we establish some sufficient conditions for Toeplitz operators and little Hankel operators on the Bergman space $A^{2}(\mathbb{D})$ to be unitary and average of unitaries.

\section{RESULTS}

\subsection{Preliminary considerations}

In this section we introduce the basic concepts and known results used in the course of our investigation. Our presentation is on the Bergman space. For more details, refer $[1,6,8,12]$.

Corollary 1 ([6]). Let $T, S \in \mathbb{B}(H)$ with $T$ invertible. If $T^{-1} S=S T^{*}$ with $0 \notin$ $\operatorname{cl}(W(S))$ and if $T$ is hyponormal, then $T$ is unitary.

Proposition 1 ([12]). For an invertible operator $T \in \mathbb{B}(H)$, the following are equivalent: (1) $T \Theta T^{-1}$ and $\|T\| \leq 1$ (2) $T$ is unitary. 
Corollary 2 ([1]). Let $T_{1} \in \mathcal{L}(H)$ be injective $w$ - hyponormal operator and $T_{2} \in$ $\mathcal{L}(H)$ be an isometry. Assume that there exists quasiaffinities $X$ and $Y$ such that $T_{1} X=X T_{2}$ and $Y T_{1}=T_{2} Y$, if either $X$ or $Y$ is compact, then $T_{1}$ and $T_{2}$ are unitary operators and unitarily equivalent.

Theorem 1 ([8]). If a normal operator $N_{1}$ is a quasi-affine transform of a normal operator $N_{2}$, then $N_{1}$ is unitarily equivalent to $N_{2}$.

Moreover, the present study has certain advanced applications which are related to the outcomes of the recent results of Padhy et al. [3] and Srivastava et al. [14].

\section{MAin Result}

\subsection{Toeplitz operators}

In this section, we present some sufficient conditions for Toeplitz operators on $A^{2}(\mathbb{D})$ to be unitary.

Theorem 2. Let $\phi$ be an essentially bounded Lebesgue measurable function on $\mathbb{D}$ such that $\|\phi\|_{\infty} \leq 1$. Then $T_{\psi}$ can be represented as an average of $n$ unitary operators, where $\psi=\left(1-\frac{1}{n}\right) \phi+\frac{1}{n}, n>2$ and $n \in \mathbb{N}$.

Proof. Since $\|\phi\|_{\infty} \leq 1$,

$$
\left\|I-T_{\frac{1+\phi}{2}}\right\|=\left\|I-\frac{I}{2}-\frac{T_{\phi}}{2}\right\|=\left\|\frac{I}{2}-\frac{T_{\phi}}{2}\right\| \leq \frac{1}{2}+\frac{1}{2}\left\|T_{\phi}\right\| \leq \frac{1+\left\|\phi_{\infty}\right\|}{2}<1 .
$$

Therefore, $T_{\frac{1+\phi}{2}}$ is invertible. Let $T_{\frac{1+\phi}{2}}$ has a polar decomposition $V P$, where $P$ is a positive operator and $V$ is a unitary operator. Therefore, $T_{\frac{1+\phi}{2}}=\frac{V\left(P_{1}+P_{2}\right)}{2}$, where $P_{1}=$ $P+i \sqrt{I-P^{2}}$ and $P_{2}=P-i \sqrt{I-P^{2}}$ are unitary operators. So, $T_{1+\phi}=V P_{1}+V P_{2}$. Similarly there are unitary operators $V_{1}, V_{2}, \ldots, V_{n-1}$ and $W_{1}, W_{2}, \ldots, W_{n-1}\left(=V_{n}\right)$ such that

$$
\begin{aligned}
T_{1+\phi} & =V_{1}+W_{1} \\
T_{1+2 \phi} & =V_{1}+W_{1}+T_{\phi} .
\end{aligned}
$$

Therefore,

$$
\begin{aligned}
T_{1+(n-1) \phi} & =V_{1}+W_{1}+T_{(n-2) \phi} \\
& =V_{1}+V_{2}+W_{2}+T_{(n-3) \phi} \\
& =\ldots \\
& =V_{1}+V_{2}+\ldots+\left(V_{n}=W_{n-1}\right) .
\end{aligned}
$$

Then,

$$
T_{\psi}=T_{\left(1-\frac{1}{n}\right) \phi+\frac{1}{n}}=\frac{1}{n} T_{1+(n-1) \phi}=\frac{1}{n}\left(V_{1}+V_{2}+\ldots+V_{n}\right) .
$$

Hence the result holds. 
Theorem 3. Let $\phi \in L^{\infty}(\mathbb{D})$ and $\phi \geq 0$. If $T_{\phi} T_{\phi-1}=0$, then $T_{\psi}$ is unitary where $\psi=2\left(\phi \circ \phi_{a}\right)-1$.

Proof. Since $\phi \geq 0, T_{\phi} \geq 0$. Then $T_{\phi}$ is self-adjoint. Further, Since $T_{\phi} T_{\phi-1}=0$, that implies $T_{\phi}\left(T_{\phi}-I\right)=0$ or $T_{\phi}^{2}=T_{\phi}$. It is well known that $U_{a} T_{\phi} U_{a}=T_{\phi \circ \phi_{a}}$ for all $a \in \mathbb{D}$. Now

$$
\begin{aligned}
T_{\psi}^{*} T_{\psi} & =T_{2\left(\phi \circ \phi_{a}\right)-1}^{*} T_{2\left(\phi \circ \phi_{a}\right)-1} \\
& =\left(2 T_{\phi \circ \phi_{a}}^{*}-I\right)\left(2 T_{\phi \circ \phi_{a}}-I\right) \\
& =\left(2\left(U_{a}^{*} T_{\phi}^{*} U_{a}^{*}\right)-I\right)\left(2\left(U_{a} T_{\phi} U_{a}\right)-I\right) \\
& =4\left(U_{a}^{*} T_{\phi}^{*} U_{a}^{*} U_{a} T_{\phi} U_{a}\right)-2 U_{a}^{*} T_{\phi}^{*} U_{a}^{*}-2 U_{a} T_{\phi} U_{a}+I \\
& =4 U_{a} T_{\phi}^{2} U_{a}-4 U_{a} T_{\phi} U_{a}+I \quad\left(\because U_{a}^{*}=U_{a}, T_{\phi}^{*}=T_{\phi} \text { and } U_{a}^{2}=I\right) \\
& =4 U_{a} T_{\phi} U_{a}-4 U_{a} T_{\phi} U_{a}+I \quad\left(\because T_{\phi}^{2}=T_{\phi}\right) \\
& =I .
\end{aligned}
$$

Similarly, one can verify that $T_{\psi} T_{\psi}^{*}=I$. Hence $T_{\psi}$ is unitary.

Theorem 4. Let $\phi$ be an essentially bounded Lebesgue measurable function on $\mathbb{D}$. If $T_{\phi}$ is an isometry and $T_{\bar{\phi} \circ \phi_{a}}$ is hyponormal for $a \in \mathbb{D}$. Then $T_{\phi}$ is unitary.

Proof. For all $a \in \mathbb{D}$, it is known that

$$
T_{\phi} U_{a}=U_{a} T_{\phi \circ \phi_{a}} .
$$

Now,

$$
U_{a} T_{\phi \circ \phi_{a}}^{*}=U_{a}\left(U_{a} T_{\phi} U_{a}\right)^{*}=U_{a} U_{a}^{*} T_{\phi}^{*} U_{a}^{*}=T_{\phi}^{*} U_{a} .
$$

Multiplying $T_{\bar{\phi}}$ on the left of equation (3.1), we obtain

$$
T_{\bar{\phi}} T_{\phi} U_{a}=T_{\bar{\phi}} U_{a} T_{\phi \circ \phi_{a}} .
$$

Since, $T_{\phi}$ is an isometry and $T_{\bar{\phi}} U_{a}=U_{a} T_{\bar{\phi} \circ \phi_{a}}$, equation (3.2) turns out to be

$$
U_{a}=T_{\bar{\phi}} U_{a} T_{\phi \circ \phi_{a}}=U_{a} T_{\bar{\phi} \circ \phi_{a}} T_{\phi \circ \phi_{a}} .
$$

Thus, $T_{\bar{\phi}_{\circ} \phi_{a}} T_{\phi \circ \phi_{a}}=I$. Further, since $T_{\bar{\phi} \circ \phi_{a}}$ is hyponormal and $T_{\bar{\phi} \circ \phi_{a}} T_{\phi \circ \phi_{a}}=I$. This implies, $T_{\bar{\phi} \circ \phi_{a}} T_{\phi_{\circ} \phi_{a}}=T_{\phi \circ \phi_{a}} T_{\bar{\phi}_{\circ} \phi_{a}}=I$. Hence, $T_{\phi}$ is unitary.

Theorem 5. Let $\phi \in L^{\infty}(\mathbb{D})$. If $T_{\phi \circ \phi_{a}}^{*^{2}} T_{\phi_{\circ} \phi_{a}}^{2}-2 T_{\phi \circ \phi_{a}}^{*} T_{\phi \circ \phi_{a}}+I=0$ and $\widetilde{T_{\phi}^{2}}(z)=1$ then $T_{\phi}$ is unitary.

Proof. It is well known that $U_{a} T_{\phi} U_{a}=T_{\phi \circ \phi_{a}}$. Given that $\widetilde{T_{\phi}^{2}}(z)=1$. Then $T_{\phi}^{2}=$ $I$ if and only if $T_{\bar{\phi}}^{2}=I$. Again $T_{\phi \circ \phi_{a}}^{*^{2}} T_{\phi \circ \phi_{a}}^{2}-2 T_{\phi \circ \phi_{a}}^{*} T_{\phi \circ \phi_{a}}+I=0$ gives $U_{a} T_{\phi}^{*^{2}} T_{\phi} U_{a}$ $2 U_{a} T_{\phi}^{*} T_{\phi} U_{a}+I=0$. Thus, $2-2 U_{a} T_{\phi}^{*} T_{\phi} U_{a}=0$ or $U_{a} T_{\phi}^{*} T_{\phi} U_{a}=I$ by using $T_{\phi}^{2}=I$ and $T_{\phi}^{*^{2}}=I$. Hence $T_{\phi}$ is isometry and invertible. Therefore $T_{\phi}$ is unitary. 
Theorem 6. If $\phi$ is a bounded harmonic function on $\mathbb{D}$ with $\|\phi\|_{\infty}=1$ and $\widetilde{T_{\phi}^{n}}(z)=1$ then $T_{\phi}$ is unitary.

Proof. Since $\phi$ is a bounded harmonic function on $\mathbb{D}$ then it follows from (Theorem 5, [4]) that $\left\|T_{\phi}\right\|=\|\phi\|_{\infty}=1$. Now $\widetilde{T_{\phi}^{n}}(z)=1$ that implies $\left\langle T_{\phi}^{n} k_{z}, k_{z}\right\rangle=1=\left\langle k_{z}, k_{z}\right\rangle$, $\forall z \in \mathbb{D}$. Thus, $T_{\phi}^{n}=I$. So $T_{\phi}$ is invertible. Then $T_{\phi}$ and $T_{\phi}^{-1}$ are power bounded and therefore similar to unitary operator (Proposition 3.8 and Corollary 1.16, [10]). Hence $\left\|T_{\phi}\right\|=1$ implies that $T_{\phi}$ is unitarily equivalent to unitary operator. Therefore $T_{\phi}$ is unitary.

Theorem 7. Let $\phi, \phi_{1}, \phi_{2} \in L^{\infty}(\mathbb{D})$ and $\|\phi\|_{\infty} \leq 1$. Then $T_{\phi}$ can be expressed as sum of three unitary operators.

Proof. Let $T_{\phi}=T_{\phi_{1}}+i T_{\phi_{2}}$ be the Cartesian decomposition of $T_{\phi}$. Since $\|\phi\|_{\infty} \leq 1$, $\left\|T_{\phi}\right\| \leq\|\phi\|_{\infty} \leq 1$. So $T_{\phi}$ is a contraction. Thus the real and imaginary parts of $T_{\phi}$ are also contractions. Let $T_{1}=\frac{1}{2}\left(T_{\phi_{1}}-\left(I-T_{\phi_{2}}^{2}\right)^{\frac{1}{2}}\right)$ and notice that $T_{1}$ is a contraction. Therefore one can define the operators $W_{1}=T_{1}+i\left(I-T_{1}^{2}\right)^{\frac{1}{2}}$ and $W_{2}=\left(I-T_{\phi_{2}}^{2}\right)^{\frac{1}{2}}+$ $i T_{\phi_{2}}$. Here $W_{1} W_{1}^{*}=W_{1}^{*} W_{1}=I$ and $W_{2} W_{2}^{*}=W_{2}^{*} W_{2}=I$. Hence $T_{\phi}=W_{1}+W_{1}^{*}+W_{2}$, which is sum of three unitary operators.

Theorem 8. Let $\phi, \phi_{1}, \phi_{2} \in L^{\infty}(\mathbb{D})$ and $T_{\phi}=T_{\phi_{1}}+i T_{\phi_{2}}$ be the Cartesian decomposition of $T_{\phi}$. Then $T_{1-\phi}$ is unitary if and only if $T_{\phi_{1}} T_{\phi_{2}}=T_{\phi_{2}} T_{\phi_{1}}$ and $T_{\phi_{1}-1}^{2}=T_{1-\phi_{2}} T_{1+\phi_{2}}$.

Proof. Suppose $T_{\phi_{1}} T_{\phi_{2}}=T_{\phi_{2}} T_{\phi_{1}}$. Then $T_{\phi} T_{\bar{\phi}}=T_{\phi} T_{\phi}=T_{\phi_{1}}^{2}+T_{\phi_{2}}^{2}$. Again, since $T_{\phi_{1}-1}^{2}=T_{1-\phi_{2}} T_{1+\phi_{2}}$ then, $\left(T_{\phi_{1}}-I\right)^{2}=\left(I-T_{\phi_{2}}\right)\left(I+T_{\phi_{2}}\right)$. So $T_{\phi} T_{\bar{\phi}}=T_{\bar{\phi}} T_{\phi}=T_{\phi+\bar{\phi}}$, it can be easily shown that $T_{1-\bar{\phi}} T_{1-\phi}=I=T_{1-\phi} T_{1-\bar{\phi}}$. Conversely, suppose $T_{1-\phi}$ is unitary. Then $T_{\phi} T_{\bar{\phi}}=T_{\bar{\phi}} T_{\phi}=T_{\phi+\bar{\phi}}$. Since $T_{\phi} T_{\bar{\phi}}=T_{\bar{\phi}} T_{\phi}$, we have $T_{\phi_{1}} T_{\phi_{2}}=T_{\phi_{2}} T_{\phi_{1}}$. Again, since $T_{\phi} T_{\bar{\phi}}=T_{\phi+\bar{\phi}}$, therefore $T_{\phi_{1}-1}^{2}=T_{1-\phi_{2}} T_{1+\phi_{2}}$.

Corollary 3. Let $\phi, \phi_{1}, \phi_{2} \in L^{\infty}(\mathbb{D})$ where $\phi_{1} \geq 0$ and $\phi_{2} \geq 0$. If $T_{\phi}=T_{\phi_{1}}+i T_{\phi_{2}}$ be the Cartesian decomposition of $T_{\phi}$ with $T_{\phi_{1}} T_{\phi_{2}}$ is p-hyponormal and $T_{\phi_{1}-1}^{2}=$ $T_{1-\phi_{2}} T_{1+\phi_{2}}$, then $T_{1-\phi}$ is unitary.

Proof. If $\phi_{1} \geq 0$ and $\phi_{2} \geq 0$, then $T_{\phi_{1}}, T_{\phi_{2}}$ are positive. Let $A=T_{\phi_{1}} T_{\phi_{2}}$, then $A T_{\phi_{1}}=T_{\phi_{1}} A^{*}$. So from [15], we get $A^{*} T_{\phi_{1}}=T_{\phi_{1}} A$, then $\left(T_{\phi_{1}} T_{\phi_{2}}\right)^{*} T_{\phi_{1}}=T_{\phi_{1}} T_{\phi_{1}} T_{\phi_{2}}$. Thus $T_{\overline{\phi_{2}}} T_{\overline{\phi_{1}}} T_{\phi_{1}}=T_{\phi_{1}}^{2} T_{\phi_{2}}$. Therefore $T_{\phi_{2}} T_{\phi_{1}}^{2}=T_{\phi_{1}}^{2} T_{\phi_{2}}$. As $T_{\phi_{1}}$ is positive, so $T_{\phi_{1}} T_{\phi_{2}}=T_{\phi_{2}} T_{\phi_{1}}$. Hence by Theorem 8 , the corollary holds.

Corollary 4. Let $\phi, \phi_{1} \in L^{\infty}(\mathbb{D})$ and $T_{\phi}=T_{\phi_{1}}+i T_{\overline{\phi_{1}}}$ be the Cartesian decomposition of $T_{\phi}$. If $T_{\phi_{1}}, T_{\bar{\phi}_{1}}$ are paranormal with $\operatorname{ker}\left(T_{\phi_{1}}\right)=\operatorname{ker}\left(T_{\overline{\phi_{1}}}\right)$ and $T_{\phi_{1}-1}^{2}=T_{1-\overline{\phi_{1}}} T_{1+\overline{\phi_{1}}}$. Then $T_{1-\phi}$ is unitary.

Proof. Since $T_{\phi_{1}}$ and $T_{\overline{\phi_{1}}}$ are paranormal with $\operatorname{ker}\left(T_{\phi_{1}}\right)=\operatorname{ker}\left(T_{\overline{\phi_{1}}}\right)$, then by (Theorem 5, [2]), $T_{\phi_{1}}$ is normal. That is $T_{\phi} T_{\bar{\phi}}=T_{\bar{\phi}} T_{\phi}$. Therefore by replacing $T_{\phi_{2}}=T_{\overline{\phi_{1}}}$ in Theorem 8, the assertion holds. 
Theorem 9. Let $\|\phi\|_{\infty} \leq 1$ for $\phi \in L^{\infty}(\mathbb{D})$ and $\left\|U_{a}-T_{\phi}\right\|<1$ for all $a \in \mathbb{D}$. If $\left\|T_{\phi}^{n}\right\|^{-1}-\left\|T_{\phi}^{-n}\right\|-\left(\left|1-\left\|T_{\phi}^{n}\right\|^{-1}\right|+\left|1-\left\|T_{\phi}^{-n}\right\|\right|\right)=0$ for all $n \in \mathbb{N}$. Then $T_{\phi}$ is unitary.

Proof. Since $\|\phi\|_{\infty} \leq 1$ and $\left\|T_{\phi}\right\| \leq 1$, supposing that $\left\|U_{a}-T_{\phi}\right\|<1$, it follows from [13] that $T_{\phi}$ is invertible. Then, $T_{\phi}^{n}$ is also invertible for $n \in \mathbb{N}$. As $T_{\phi}$ is a contraction, $\left\|T_{\phi}^{n}\right\|^{-1} \geq 1$. Thus, $1 \leq\left\|T_{\phi}^{-n}\right\|\left\|T_{\phi}^{n}\right\|$ and therefore, $\left\|T_{\phi}^{-n}\right\| \geq 1$. Since, $\left\|T_{\phi}^{n}\right\|^{-1}-\left\|T_{\phi}^{-n}\right\|=\left(\left|1-\left\|T_{\phi}^{n}\right\|^{-1}\right|+\left|1-\left\|T_{\phi}^{-n}\right\|\right|\right)$, this implies $\left\|T_{\phi}^{-n}\right\|=1$. Therefore, $T_{\phi}^{n}$ is unitary on $A^{2}(\mathbb{D})$. Further, assuming that $\left\|T_{\phi}\right\| \leq 1$ and $T_{\phi}^{n}$ is an isometry, $T_{\phi}$ is unitary. In particular, if $\left\|T_{\phi}\right\| \leq 1$ then $\left\|T_{\phi}^{n-1}\right\| \leq 1$. This implies that $T_{\bar{\phi}}^{n-1} T_{\phi}^{n-1} \leq$ $I$. Therefore, $I \geq T_{\bar{\phi}} T_{\phi} \geq T_{\bar{\phi}}\left(T_{\bar{\phi}}^{n-1} T_{\phi}^{n-1}\right) T_{\phi}=T_{\bar{\phi}}^{n} T_{\phi}^{n}=I$. Hence, $T_{\phi}$ is an isometry. Further, if $\left\|T_{\phi}\right\| \leq 1$ and $T_{\bar{\phi}}^{n}$ is an isometry, $T_{\bar{\phi}}$ is an isometry. So, $\left\|T_{\phi}\right\| \leq 1$ and $T_{\phi}^{n}$ is unitary, it clearly implies that $T_{\phi}$ is a unitary operator.

Theorem 10. Let $\phi$ be a positive essentially bounded Lebesgue measurable function with $\|\phi\|_{\infty} \leq 1$. Then $\mp T_{\phi} \pm i \sqrt{I-T_{\phi}^{2}}$ are unitary operators on $A^{2}(\mathbb{D})$.

Proof. As $\phi$ is positive, $T_{\phi}$ is positive on $A^{2}(\mathbb{D})$ and since $\|\phi\|_{\infty} \leq 1,\left\|T_{\phi}\right\| \leq 1$. Therefore, $I-T_{\phi}^{2}$ is a positive operator on $A^{2}(\mathbb{D})$. Let's define $U_{1}=T_{\phi}+i \sqrt{I-T_{\phi}^{2}}$, $U_{2}=T_{\phi}-i \sqrt{I-T_{\phi}^{2}}, U_{3}=-T_{\phi}+i \sqrt{I-T_{\phi}^{2}}$ and $U_{4}=-T_{\phi}-i \sqrt{I-T_{\phi}^{2}}$. One can easily observe that, $U_{1}^{*}=U_{2}, U_{3}^{*}=U_{4}$. Hence, $U_{1}^{*} U_{1}=U_{1} U_{1}^{*}=I, U_{2}^{*} U_{2}=U_{2} U_{2}^{*}=$ $I, U_{3}^{*} U_{3}=U_{3} U_{3}^{*}=I$ and $U_{4}^{*} U_{4}=U_{4} U_{4}^{*}=I$. Therefore, $U_{1}, U_{2}, U_{3}$ and $U_{4}$ are unitary operators on $A^{2}(\mathbb{D})$.

Proposition 2. Let $\phi \in L^{\infty}(\mathbb{D})$. If $\widetilde{T_{\phi}^{2}}(z)=1$ then $T_{\phi}$ is unitarily equivalent with $T_{\bar{\phi}}$.

Proof. Let $T_{\phi}=U P$ be the polar decomposition of $T$ where $U$ is partial isometry and $P$ is the positive operator. Since positive operators are self-adjoint, so $P^{*}=P$. Further since $\widetilde{T_{\phi}^{2}}(z)=1,\left\langle T_{\phi}^{2} k_{z}, k_{z}\right\rangle=1=\left\langle k_{z}, k_{z}\right\rangle, \forall z \in \mathbb{D}$. That implies $T_{\phi}^{2}=I$ and therefore $T_{\phi}$ is invertible. Hence the partial isometry $U$ will become a unitary operator $U_{a}$ (say) $\forall a \in \mathbb{D}$. Thus $T_{\phi}=U_{a} P$ where $U_{a}=U_{a}^{*}=U_{a}^{-1}$. Then

$$
U_{a} T_{\phi} U_{a}=U_{a} U_{a} P U_{a}=P U_{a}=T_{\phi} .
$$

Hence $T_{\phi}$ is unitarily equivalent with $T_{\bar{\phi}}$.

Theorem 11. Let $\phi \in L^{\infty}(\mathbb{D})$ and $\phi \geq 0$. Then $T_{\phi}$ is unitary if and only if $\widetilde{T_{\phi}^{2}}(z)=1$.

Proof. Let $\phi \geq 0$, that implies $T_{\phi} \geq 0$. Since positive operators are self adjoint, $T_{\phi}^{*}=T_{\phi}$. Given $\widetilde{T_{\phi}^{2}}(z)=1$, so we get $T_{\phi}^{2}=I$. Now

$$
T_{\phi}^{*} T_{\phi}=T_{\phi}^{2}=I=T_{\phi} T_{\phi}^{*} .
$$


So $T_{\phi}$ is unitary. Conversely suppose $T_{\phi}$ is unitary, then $T_{\phi}^{2}=I$. Thus $\left\langle T_{\phi}^{2} f, g\right\rangle=$ $\langle f, g\rangle \forall f, g \in A^{2}(\mathbb{D})$. If $f=g=k_{z}, \forall z \in \mathbb{D}$, then $\widetilde{T_{\phi}^{2}}(z)=1$.

\subsection{Little Hankle operators}

In this section, we discuss some sufficient conditions for little Hankle operators on $A^{2}(\mathbb{D})$ to be unitary.

Theorem 12. Let $\phi \in L^{\infty}(\mathbb{D})$. If $S_{\phi}$ is power bounded and bounded below with $\left\|S_{\phi}^{2}\right\|^{2}+1=2\left\|S_{\phi}\right\|^{2}$ then $S_{\phi}$ is unitary.

Proof. Given that

$$
\left\|S_{\phi}^{2}\right\|^{2}+1=2\left\|S_{\phi}\right\|^{2} .
$$

We have to show $S_{\phi}^{n}$ will satisfy the equation (3.4). We will prove this by method of induction. Now the above equation is true for $n=1$. Let us assume that equation (3.4) is true for $n=k$, that is $\left\|S_{\phi}^{2 k}\right\|^{2}+1=2\left\|S_{\phi}^{k}\right\|^{2}$. This implies

$$
S_{\phi}^{*^{2 k}} S_{\phi}^{2 k}+I=2 S_{\phi}^{*^{k}} S_{\phi}^{k} .
$$

Then we will prove that equation (3.4) is true for $n=k+1$. Now

$$
\begin{aligned}
S_{\phi}^{*^{2(k+1)}} S_{\phi}^{2(k+1)}+I-2 S_{\phi}^{k^{k+1}} S_{\phi}^{k+1} & =S_{\phi}^{*^{2}}\left(S_{\phi}^{*^{2 k}} S_{\phi}^{2 k}\right) S_{\phi}^{2}+I-2 S_{\phi}^{*^{k+1}} S_{\phi}^{k+1} \\
& =S_{\phi}^{*^{2}}\left(2 S_{\phi}^{*^{k}} S_{\phi}^{k}-I\right) S_{\phi}^{2}+I-2 S_{\phi}^{*^{k+1}} S_{\phi}^{k+1} \\
& =2 S_{\phi}^{*^{k+2}} S_{\phi}^{k+2}-S_{\phi}^{*^{2}} S_{\phi}^{2}+I-2 S_{\phi}^{*^{k+1}} S_{\phi}^{k+1} \\
& =2 S_{\phi}^{*^{k}}\left(S_{\phi}^{*^{2}} S_{\phi}^{2}-S_{\phi}^{*} S_{\phi}\right) S_{\phi}^{k}-S_{\phi}^{*^{2}} S_{\phi}^{2}+I \\
& =2 S_{\phi}^{*^{k}}\left(S_{\phi}^{*} S_{\phi}-I\right) S_{\phi}^{k}-S_{\phi}^{*^{2}} S_{\phi}^{2}+I \quad(b y(3.4)) \\
& =2 S_{\phi}^{*^{k+1}} S_{\phi}^{k+1}-2 S_{\phi}^{*^{k}} S_{\phi}^{k}-S_{\phi}^{*^{2}} S_{\phi}^{2}+I \\
& =2 S_{\phi}^{*^{k-1}}\left(S_{\phi}^{*^{2}} S_{\phi}^{2}-S_{\phi}^{*} S_{\phi}\right) S_{\phi}^{k-1}-S_{\phi}^{*^{2}} S_{\phi}^{2}+I \\
& =2 S_{\phi}^{*^{k-1}}\left(S_{\phi}^{*} S_{\phi}-I\right) S_{\phi}^{k-1}-S_{\phi}^{*^{2}} S_{\phi}^{2}+I \\
& =2 S_{\phi}^{*^{k}} S_{\phi}^{k}-2 S_{\phi}^{*^{k-1}} S_{\phi}^{k-1}-S_{\phi}^{*^{2}} S_{\phi}^{2}+I
\end{aligned}
$$

continuing in this fashion

$$
\begin{aligned}
& =2 S_{\phi}^{*^{2}} S_{\phi}^{2}-2 S_{\phi}^{*} S_{\phi}-S_{\phi}^{*^{2}} S_{\phi}^{2}+I \\
& =S_{\phi}^{*^{2}} S_{\phi}^{2}-2 S_{\phi}^{*} S_{\phi}+I \\
& =0
\end{aligned}
$$


where the second equality follows from (3.5). Hence $S_{\phi}^{n}$ is satisfying equation (3.4) that is, $\left\|S_{\phi}^{2 n}\right\|^{2}+1=2\left\|S_{\phi}^{n}\right\|^{2}$. Therefore,

$$
S_{\phi}^{*^{2 n}} S_{\phi}^{2 n}-2 S_{\phi}^{*^{n}} S_{\phi}^{n}+I=0
$$

Again from equation (3.4),

$$
\left\|S_{\phi}^{2}\right\|^{2}=2\left\|S_{\phi}\right\|^{2}-1
$$

So

$$
\left\|S_{\phi}^{4}\right\|^{2}=2\left\|S_{\phi}^{2}\right\|^{2}-1=2\left(2\left\|S_{\phi}\right\|^{2}-1\right)-1=4\left\|S_{\phi}\right\|^{2}-3 .
$$

Again,

$$
\left\|S_{\phi}^{8}\right\|^{2}=2\left\|S_{\phi}^{4}\right\|^{2}-1=2\left(4\left\|S_{\phi}\right\|^{2}-3\right)-1=8\left\|S_{\phi}\right\|^{2}-7 .
$$

In general, we get

$$
\left\|S_{\phi}^{2^{n}}\right\|^{2}=2^{n}\left\|S_{\phi}\right\|^{2}-\left(2^{n}-1\right)
$$

for every $n \in \mathbb{N}$. Further, since $S_{\phi}$ is power bounded, so $\exists$ a positive real number $M$ such that

$$
\left\|S_{\phi}^{n}\right\| \leq M \quad \text { for } n=1,2,3 \ldots .
$$

Thus, equation (3.7) and (3.8) will provide $M^{2} \geq\left\|S_{\phi}^{2^{n}}\right\|^{2}=2^{n}\left\|S_{\phi}\right\|^{2}-\left(2^{n}-1\right)$. This implies, $\frac{M^{2}}{2^{n}} \geq\left\|S_{\phi}\right\|^{2}-1+2^{-n} \geq 0$. As $n \rightarrow \infty$, we get $\left\|S_{\phi}\right\|^{2}=1$. Therefore,

$$
S_{\phi}^{*} S_{\phi}=I \text {. }
$$

Since any isometry is one-to-one, $\operatorname{ker}\left(S_{\phi}\right)=\{0\}$. It follows from ([5], Lemma-2.1) that $\operatorname{ker}\left(S_{\phi}^{*}\right)=\{0\}$. It is well known that $\overline{\operatorname{Range}\left(S_{\phi}\right)}=\operatorname{ker}\left(S_{\phi}^{*}\right)^{\perp}=\{0\}^{\perp}=A^{2}(\mathbb{D})$. That implies Range $\left(S_{\phi}\right)$ is dense in $A^{2}(\mathbb{D})$. Since $S_{\phi}$ is bounded below and has dance range, then by [7], $S_{\phi}$ is invertible and Range $\left(S_{\phi}\right)=A^{2}(\mathbb{D})$. Let $S_{\phi}=U P$ be the polar decomposition of $S_{\phi}$ where $U$ is the partial isometry and $P$ is the positive operator. Since $S_{\phi}$ is invertible, so the partial isometry $U$ can be extended to a unitary operator $V$ (say). Therefore, $S_{\phi}=V P$. It can be easily shown that $S_{\phi}=V N$, where $N$ is a normal operator. Hence,

$$
S_{\phi} S_{\phi}^{*}=V S_{\phi}^{*} S_{\phi} V^{*}=I \text {. }
$$

Therefore, $S_{\phi}$ is unitary.

Corollary 5. Let $\phi \in L^{\infty}(\mathbb{D})$ and $\|\phi\|_{\infty} \leq 1$. If $\widetilde{\left(S_{\phi}^{*} S_{\phi}\right)}(z) \geq 1$ and $S_{\phi}$ is bounded below. Then $S_{\phi}$ is unitary.

Proof. Now for any $f \in A^{2}(\mathbb{D})$,

$$
\left\|S_{\phi} f\right\| \leq\left\|S_{\phi}\right\|\|f\| \leq\|\phi\|_{\infty}\|f\| \leq\|f\| .
$$

Thus,

$$
\left\|S_{\phi}\right\|^{2} \leq\|f\|^{2} \Rightarrow\left\langle S_{\phi} f, S_{\phi} f\right\rangle \leq\langle f, f\rangle \Rightarrow S_{\phi}^{*} S_{\phi} \leq I .
$$


Again, since $\widetilde{\left(S_{\phi}^{*} S_{\phi}\right)}(z) \geq 1,\left\langle S_{\phi} k_{z}, S_{\phi} k_{z}\right\rangle \geq 1=\left\|k_{z}\right\|^{2},\left\langle k_{z}, k_{z}\right\rangle \leq\left\langle S_{\phi}^{*} S_{\phi} k_{z}, k_{z}\right\rangle$ and also $I \leq S_{\phi}^{*} S_{\phi}$. Hence, $S_{\phi}$ is isometry. Thus, the corollary follows from Theorem 12 .

Corollary 6. Let $\phi \in L^{\infty}(\mathbb{D})$. If $\left\|S_{\phi}^{2}\right\|^{2}+1=2\left\|S_{\phi}\right\|^{2}$ and $\widetilde{S_{\phi}^{2}}(z)=1$. Then $S_{\phi}$ is unitary.

Proof. Given that $\widetilde{S_{\phi}^{2}}(z)=1$. Then $\left\langle S_{\phi}^{2} k_{z}, k_{z}\right\rangle=1=\left\|k_{z}\right\|^{2}$. That implies $\left\langle S_{\phi}^{2} k_{z}, k_{z}\right\rangle=$ $\left\langle k_{z}, k_{z}\right\rangle$. That is $S_{\phi}^{2}=I$. It is easy to verify that $S_{\phi}^{2}=I$ if and only if $S_{\phi}^{*^{2}}=I$. Since $\left\|S_{\phi}^{2}\right\|^{2}+1-2\left\|S_{\phi}\right\|^{2}=0$ then $S_{\phi}^{*^{2}} S_{\phi}^{2}+I-2 S_{\phi}^{*} S_{\phi}=0$ and an easy computation demonstrates $2 I-2 S_{\phi}^{*} S_{\phi}=0$ or $I=S_{\phi}^{*} S_{\phi}$. Thus $S_{\phi}$ is isometry. Further, Since $S_{\phi}^{2}=I$ then $S_{\phi}$ is right invertible as well as left invertible and hence $S_{\phi}$ is invertible. Therefore, the corollary follows from Theorem 12 .

Corollary 7. Let $\phi \in L^{\infty}(\mathbb{D})$. If $S_{\phi}$ is normaloid and $\widetilde{S_{\phi}^{n}}(z)=1$, then $S_{\phi}$ is unitary.

Proof. It is well known that $\left\|S_{\phi}^{n}\right\| \leq\left\|S_{\phi}\right\|^{n}$, for $n \in \mathbb{N}$. Since $\widetilde{S_{\phi}^{n}}(z)=1$, we have that $\left\langle S_{\phi}^{n} k_{z}, k_{z}\right\rangle=1=\left\|k_{z}\right\|^{2}=\left\langle k_{z}, k_{z}\right\rangle$. That is $S_{\phi}^{n}=I$. Again, since $S_{\phi}$ is normaloid, then $\left\|S_{\phi}^{n}\right\|=\left\|S_{\phi}\right\|^{n}=\left\|S_{\phi}\right\|$. Since $S_{\phi}^{n}=I$, then it is obvious that $1=\left\|S_{\phi}^{n}\right\|=\left\|S_{\phi}\right\|^{n}=$ $\left\|S_{\phi}\right\|$. Thus $S_{\phi}$ is isometry and $S_{\phi}^{2}=I$. Therefore, the corollary follows from Corollary 6.

Theorem 13. Let $\phi \in L^{\infty}(\mathbb{D})$ and $T_{\phi}$, $S_{\phi}^{*}$ are p-hyponormal operators with $T_{\phi} X=$ $X S_{\phi}$ where $X: A^{2}(\mathbb{D}) \rightarrow A^{2}(\mathbb{D})$ such that $\operatorname{ker}(X)=\{0\}$ and $\overline{\text { Range }(X)}=A^{2}(\mathbb{D})$. If $T_{1-\phi}$ is unitary then $S_{1-\phi}$ is unitary.

Proof. Since $T_{\phi}, S_{\phi}^{*}$ are p-hyponormal operators with $T_{\phi} X=X S_{\phi}$ where $X: A^{2}(\mathbb{D})$ $\rightarrow A^{2}(\mathbb{D})$ such that $\operatorname{ker}(X)=\{0\}$ and $\overline{\operatorname{Range}(X)}=A^{2}(\mathbb{D})$ then from [9], $S_{\phi}$ is unitarily equivalent to $T_{\phi}$. That is $S_{\phi}=V^{*} T_{\phi} V$ where $V$ is unitary. As $T_{1-\phi}=I-T_{\phi}$ is unitary, so $T_{\bar{\phi}} T_{\phi}=T_{\phi+\bar{\phi}}=T_{\phi} T_{\bar{\phi}}$. Thus $S_{\phi}+S_{\phi}^{*}=V^{*} T_{\phi+\bar{\phi}} V$. Now

$$
\begin{aligned}
S_{1-\phi}^{*} S_{1-\phi} & =\left(I-S_{\phi}\right)^{*}\left(I-S_{\phi}\right) \\
& =S_{\phi}^{*} S_{\phi}-S_{\phi}^{*}-S_{\phi}+I \\
& =V^{*} T_{\phi}^{*} T_{\phi} V-S_{\phi}^{*}-S_{\phi}+I \\
& =V^{*} T_{\phi}^{*} T_{\phi} V-V^{*} T_{\phi+\bar{\phi}} V+I=I .
\end{aligned}
$$

Similarly, we have $S_{1-\phi} S_{1-\phi}^{*}=I$. Hence the assertion holds.

\section{ACKNOWLEDGEMENT}

The help of the librarian, central library VSSUT is acknowledged with thanks for providing permission to access through different sources. Moreover we express our gratitude to the esteemed reviewer for the valuable suggestions to enhance the quality of the paper. 


\section{REFERENCES}

[1] M. H. M. Rashid, M. S. M. Noorani, and A. S. Saari, " On the spectra of some non-normal operators," Bull. Malays. Math. Sci. Soc, vol. 31, pp. 135-143, 2008.

[2] T. Ando, "Operators with norm condition." Acta Sci. Math. (Szeged), vol. 33, pp. 169-178, 1972.

[3] C. Padhy, P. K. Jena, and S. K. Paikray, "Aluthge transform of operators on the Bergman space," Arab. J. Math., vol. 8, pp. 1-11, 2019, doi: 10.1007/s40065-019-00272-y.

[4] N. Das, “ Norm of Toeplitz operators on the Bergman space," Indian J. Pure Appl. Math, vol. 33, pp. 255-267, 2002.

[5] N. Das and P. K. Jena, “ On the range and kernel Of Toeplitz and little Hankel operators." Methods Funct. Anal. Topology, vol. 19, pp. 55-67, 2013.

[6] C. R. Deprima, “ Remarks on "Operators with inverses similar to their adjoints"," Porc. Amer. Math. Soc., vol. 43, pp. 478-480, 1974, doi: 10.1090/S0002-9939-1974-0331080-4.

[7] R. G. Douglas, Banach Algebra Techniques in Operator Theory. New York: Academic Press, 1972.

[8] H.L.Wang, “ A note on uniform operators,” Porc. Amer. Math. Soc., vol. 96, pp. 643-646, 1986, doi: $10.2307 / 2045921$.

[9] I. H. Kim, “ The Fuglede-Putnam theorem for $(P, K)$ - quasihyponormal operators," J. Inequal Appl., vol. 2006, pp. 1-7, 2004, doi: 10.1155/JIA/2006/47481.

[10] C. S. Kubrusly, An introduction to models and decompositions in operator theory. Boston: Birkh'auser, 1997.

[11] K.Zhu, Operator Theory in Function Spaces. New York: Marcel Dekker, 1990.

[12] I. S. Othman, “ Nearly equivalent operators,” Mathematica Bohemica, vol. 121, pp. 133-141, 1996.

[13] C. S. Lin, “ The unilateral shift and a norm equality for bounded linear operators," Porc. Amer. Math. Soc., vol. 127, pp. 1693-1696, 1999.

[14] H. M. Srivastava, Q. Z. Ahmad, N. Khan, and N. Khanand B.Khan, "Hankel and Toeplitz determinant for a subclass of $q$ starlike functions associated with a general conic domain," Mathematics, vol. 7, pp. 1-15, 2019.

[15] A. Uchiyama and K. Tanahashi, “ Fuglede-Putnam's theorem for p-hyponormal or log-hyponormal operators," Glasgow Math. J., vol. 44, pp. 397-410, 2002, doi: 10.1017/S00170895020201399.

Authors' addresses

C. Padhy

Veer Surendra Sai University of Technology, Dep. of Mathematics, Burla-768018, Odisha, India

E-mail address: chinmayee.padhy83@gmail.com

\section{P. K. Jena}

(Corresponding author) Berhampur University, Bhanjabihar, P. G. Dep. of Mathematics, Berhampur, Ganjam-760007, Odisha, India

E-mail address: pabitramath@gmail.com

\section{S. K. Paikray}

Veer Surendra Sai University of Technology, Dep. of Mathematics, Burla-768018, Odisha, India

E-mail address: skpaikray mathevssut.ac. in 